\title{
Targeted hepatitis B vaccination - a cost effective immunisation strategy for the UK?
}

\author{
John R Williams, D James Nokes, Roy M Anderson
}

\begin{abstract}
Objective - To compare the potential cost effectiveness of vaccination against hepatitis $B$ virus (HBV) targeted at genitourinary clinic (GU) attendees with that of universal infant vaccination.

Design - A mathematical model of sexual and perinatal transmission of $\mathrm{HBV}$ was used to compare the effectiveness among heterosexual and homosexual populations of programmes of mass infant vaccination and targeted immunisation of genitourinary medicine (GU) clinic attendees. Each was applied to $90 \%$ of the eligible population with differing assumptions about rates of compliance and seroconversion - problems of delivery (obtaining high compliance) was considered a significant drawback of targeted vaccination. Observed relationships between GU clinic attendance and sex partner change rates for heterosexuals and for homosexuals were used to define the rates of vaccination uptake within sexual activity risk groups.

Setting - England and Wales.
\end{abstract}

Results - Model results showed that for heterosexuals universal infant vaccination became more effective than clinic based vaccination only approximately 40 years after the start of the programme and that the predicted cost effectiveness of GU clinic vaccination was greater at all times. For homosexuals, clinic vaccination was always more effective over the time frame considered, but by 50 years if it were carried out without prior screening it had become appreciably less cost effective than a mass infant programme. With prior screening in GU clinics this cost effectiveness deficit was only marginal.

Conclusions - Targeted vaccination might have a much greater potential than is realised at present, particularly if it were possible to improve compliance of clinic attendees. A fuller comparison between mass infant and targeted vaccination must await the specific inclusion in the model of other risk groups such as intravenous drug users. An important determinant of the relative merits of the two approaches is the relationship between rates of attendance and of changing sexual partners. Further research on this is required.

( $\mathcal{F}$ Epidemiol Community Health 1996;50:667-673)

The World Health Organization (WHO) recommends that all countries should introduce universal immunisation against hepatitis B virus (HBV) by 1997 and that countries with a prevalence of $\mathrm{HBV}$ carriers that is less than $2 \%$ should consider vaccinating adolescents as an alternative or in addition to infant vaccination. ${ }^{1}$ In the European Community, Italy, Spain, Portugal, and France have either implemented universal infant or adolescent immunisation or are in the final stages of doing so, ${ }^{2}$ and Greece is in the process of moving towards the practice of universal vaccination. ${ }^{3}$ Other countries in north western Europe and Scandinavia practise various forms of selective immunisation more or less nationally applied - for example, antenatal screening followed by vaccination of those identified as being at risk, vaccination of those at high risk because of their behaviour, such as sexually transmitted disease (STD) clinic attendees and intravenous drug users, and vaccination of those who are at risk because of their work. ${ }^{4}$ In many of these countries, however, irrespective of countryspecific epidemiological status, there is increased support for universal infant or adolescent immunisation as a means of achieving the goal of $\mathrm{HBV}$ eradication throughout the European region. WHO and the pharmaceutical industry are promoting this goal. ${ }^{56}$

Within Europe, universal vaccination was first adopted in the southern countries, where $\mathrm{HBV}$ is a far more serious problem than in northern regions. In the United Kingdom, however, a decision about implementing universal infant or adolescent immunisation is unlikely to be made solely on the grounds of WHO recommendations or as a result of the approaches adopted in other European countries. Rather it will be made on grounds of affordability, cost effectiveness in relation to other demands on the health services, and after determining whether the burden of disease and mortality induced by $\mathrm{HBV}$ in the UK is significant (both in its own right and relative to other causes of morbidity and mortality). Advocates of universal HBV immunisation in the UK must therefore argue their case taking due account of the issue of the cost effectiveness in the UK of the different programme options. ${ }^{78}$ In particular, it is important to provide quantitative details of the predicted effectiveness of universal infant (or adolescent) immunisation in comparison with nationally promoted selective immunisation programmes specifically targeted at individuals at risk, such as those who attend genitourinary (GU) clinics or intravenous drug user centres. For example, at present GU clinic vaccination is operated on a somewhat piecemeal basis with a good deal of variation in uptake and coverage between 
clinics $^{910}$ and there may be much scope for increasing its impact by a uniformly applied policy of vaccinating all those who attend.

In much work on cost effectiveness, it has become the practice to base analysis on epidemiological data derived from randomised controlled trials. However, in assessing the benefits of immunisation against an infectious disease it is insufficient to assume a constant risk of infection and to extrapolate the effect of vaccinating a relatively small group of trial participants in an attempt to predict the impact of a programme vaccinating a much larger proportion of the general population. To do so ignores the importance of the level of immunity in the population in controlling the spread of infectious disease. Immunisation of a group of individuals not only protects those individuals, but also reduces the risk or force of infection for people who are unvaccinated because the pool of potentially infectious individuals is now smaller. The impact on rates of infection of this "herd immunity effect" changes nonlinearly as the overall proportion of the population which is vaccinated increases, and this undermines attempts to base predictions on linear extrapolation of results based on controlled randomised trials. An additional drawback of the traditional approach is seen when considering infections such as HBV where several decades may elapse between infection and the risk against which vaccination is primarily aimed - that is, severe chronic disease. In these circumstances, it becomes difficult to countenance delaying the implementation of a vaccination programme until trial results are known. Here we have avoided these drawbacks by adopting an alternative approach using a mathematical model which allows us to make projections of the impact of vaccination programmes over time, and which takes full account of the non-linearities that arise from the operation of herd immunity.

In previously published work, ${ }^{8}$ a mathematical model was used to consider the effects of vaccination in the context of two major risk groups - homosexuals and heterosexuals. Our premises were that sexual transmission of the virus within heterosexual and homosexual communities implies that people who change sexual partners frequently are disproportionately important to the spread of $\mathrm{HBV}$ infection and that the frequency of attendance at GU clinics is positively associated with the partner change rate. As described in the earlier work, ${ }^{8}$ data from the national survey of sexual attitudes and lifestyles (NATSSAL), which was funded by the Wellcome Trust, ${ }^{12}$ was used to provide estimates of rates of sexual partner change and frequency of attendance at GU clinics. Here we use the framework provided by this earlier study $^{8}$ to examine the potential of selective vaccination targeted at $\mathrm{GU}$ clinic attendees to significantly reduce $\mathrm{HBV}$ transmission in the population as a whole and we explore the potential merits of a GU clinic based immunisation policy relative to one of infant immunisation. We examine the effects of vaccination programmes reaching $90 \%$ of the intended recipient population, with rates of compliance and seroconversion reflecting possible UK values. In our earlier work we used numbers of vaccine doses delivered as an indicator of cost. This is now developed by applying to our analysis of cost effectiveness monetary costings based on data for England and Wales and described in the recently published work of Mangtani et al. ${ }^{7}$ Our results are presented in terms of the relationship between the relative effectiveness and the cost effectiveness of policies in reducing numbers of $\mathrm{HBV}$ carriers, as both measures are required for realistic comparisons.

\section{Methods}

MODEL STRUCTURE

The structure of the model is described in detail in Williams et al. ${ }^{8}$ It is a deterministic model of the kind widely used in exploring the transmission dynamics of sexually transmitted disease. ${ }^{13}$ The model has six compartments containing populations who are: susceptible to infection; latently, acutely, and chronically infected; recovered and immune after infection; and immune as a result of vaccination (fig 1 ). The model population is fully age structured. Because the distribution of different levels of sexual activity (that is, rates of partner acquisition) in the population is highly skewed with a high variance, ${ }^{12}$ mean levels of activity do not adequately reflect the HBV transmission potential of those with high levels of partner change. These people are likely to be very important to the spread of this sexually transmitted disease. To overcome this, the model population also has been stratified in relation to rates of acquisition of sexual partners. There were six different activity groups and rates of partner change also vary by age. HBV transmission in the model occurs through sexual contact or at birth; and there are separate versions for heterosexual and homosexual populations (transmission via injecting drug use was not considered). At each time point the rate at which susceptible individuals are infected depended on the transmission risks, numbers of acutely and chronically infected individuals, their rates of partner change and fertility, and their distribution through the heterogeneous model population (tables 1 and 2). Once infected, individuals move at constant rates (table 1) from latent to acute infection, from acute to either chronic infection or recovery, and from chronic infection to recovery (fig 1).

Several different vaccination programmes are allowed by the model, two of which are considered here - mass vaccination at birth and a programme targeted at individuals attending GU clinics.

\section{DATA SOURCES AND ANALYSIS}

Key parameter values are set out in table 1 . Their estimation is described elsewhere ${ }^{8}$ as is the estimation of rates of partner acquisition for the sexual activity classes (using raw data originating from the NATSSAL ${ }^{12}$ ) and the way in which non-linear functional relationships were established between rates of partner ac- 


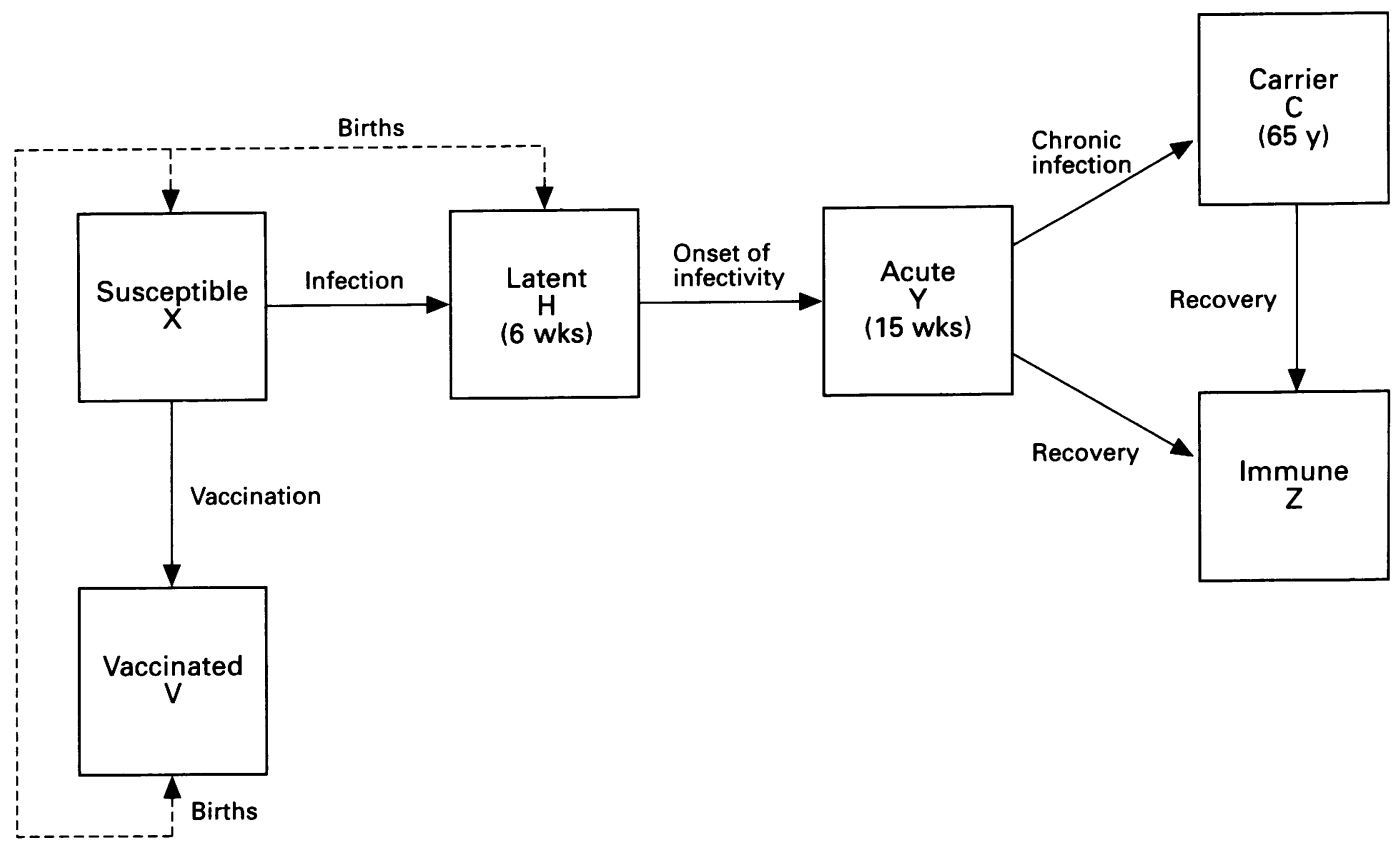

Figure 1 Flow chart showing the six compartments of the hepatitis $B$ virus transmission dynamics model. ${ }^{8}$ Births (broken lines) may occur in susceptible, latently infected, or vaccinated compartments. There are no deaths before age 60, after which age individuals play no further part in transmission. The mean duration of stay in latent, acute, and carrier compartments is shown in table 1.

quisition by heterosexuals and homosexuals and their rates of attendance at GU clinics (also using NATSSAL ${ }^{12}$ data). The salient features of these relationships are that rates of partner acquisition for heterosexuals are directly associated with rates of clinic attendance of between less than once per lifetime and once in 20 years depending on activity class (table 2 ). Attendance rates for homosexuals are about an order of magnitude higher (from less than once in 30 years to once in 3 years). A very steep initial increase in the attendance/partnership rate relationship for homosexuals accounts for their much higher rate of attendance overall. However, a high level of variance was recorded

Table 1 Model parameters relating to infection, fertility, and vaccination

\begin{tabular}{|c|c|c|c|}
\hline Parameter & Heterosexual & Homosexual & Both \\
\hline \multicolumn{4}{|c|}{ Transmission risk (/y/partnership): } \\
\hline Acute infection & 0.33 & 0.46 & \\
\hline Carrier & 0.25 & 0.30 & \\
\hline \multicolumn{4}{|c|}{ Average duration of time within infection states (wk): } \\
\hline Incubation & & & 6 \\
\hline Acute infection & & & 15 \\
\hline Carrier state & & & 65 \\
\hline \multicolumn{4}{|c|}{ Proportion of acutely infected who become carriers: } \\
\hline Infant & & & 0.885 \\
\hline Adult & & & 0.1 \\
\hline \multicolumn{4}{|c|}{ Proportion of babies born infected: } \\
\hline Acute mother & & & 0.724 \\
\hline Carrier mother & & & 0.115 \\
\hline
\end{tabular}

${ }^{*}$ See Williams $e t a l^{\beta}$ for full details. in the data, and it is clear that more studies are needed to examine this variability. Table 2 also shows mean rates of partner acquisition for each of the model's six sexual activity classes (averaged over all age classes), and the much higher partner rates of homosexuals than heterosexuals.

\section{OUTCOME MEASURES}

It is quite possible for vaccination to be highly cost effective without being highly effective in reducing the numbers of cases (as with vaccination of neonates known to be at risk of infection from their mothers ${ }^{8}$ ), so it is important to look at both factors when considering the advantages of different programmes. If the goal of a vaccination programme is to interrupt the chain of transmission in the population at large, its effectiveness must be measured in terms of numbers of cases of chronic carriers of disease prevented, as their number makes a much larger contribution to the net force of infection within the population than that of acutely infected individuals. Although individual prophylaxis by vaccination is rapid, carrier state duration is measured in decades and it takes many years of mass or targeted vaccination for transmission in the population to be markedly affected. In-

Table 2 Rates of partner acquisition and clinic attendance averaged across all age groups ${ }^{812}$

\begin{tabular}{|c|c|c|c|c|c|c|c|c|}
\hline \multirow[b]{2}{*}{$\begin{array}{l}\text { Activity } \\
\text { class }\end{array}$} & \multicolumn{4}{|c|}{ Heterosexuals } & \multicolumn{4}{|c|}{ Homosexuals } \\
\hline & $\begin{array}{l}\text { Partners } \\
\text { (ly) }\end{array}$ & $\begin{array}{l}\text { Proportion } \\
\text { in class }\end{array}$ & $\begin{array}{l}\text { Rate of clinic } \\
\text { attendance } \\
(\mid y)\end{array}$ & $\begin{array}{l}\text { Mean interval } \\
\text { between } \\
\text { attendances }\end{array}$ & $\begin{array}{l}\text { Partners } \\
(\mid y)\end{array}$ & $\begin{array}{l}\text { Proportion } \\
\text { in class }\end{array}$ & $\begin{array}{l}\text { Rate of clinic } \\
\text { attendance } \\
\text { (/y) }\end{array}$ & $\begin{array}{l}\text { Mean interval } \\
\text { between } \\
\text { attendances (y) }\end{array}$ \\
\hline $\begin{array}{l}1 \\
2 \\
3 \\
4 \\
5 \\
6\end{array}$ & $\begin{array}{l}0.025 \\
0.21 \\
0.8 \\
2.5 \\
6.1 \\
8.9\end{array}$ & $\begin{array}{l}0.273 \\
0.286 \\
0.303 \\
0.132 \\
0.005 \\
0.001\end{array}$ & $\begin{array}{l}0.0004 \\
0.0021 \\
0.0061 \\
0.0152 \\
0.0309 \\
0.0419\end{array}$ & $\begin{array}{l}>\text { lifetime } \\
>\text { lifetime } \\
>\text { lifetime } \\
\sim 66 \mathrm{y} \\
\sim 32 \mathrm{y} \\
\sim 24 \mathrm{y}\end{array}$ & $\begin{array}{c}0.16 \\
1.55 \\
4.6 \\
9.2 \\
17.0 \\
28.1\end{array}$ & $\begin{array}{l}0.451 \\
0.353 \\
0.125 \\
0.06 \\
0.01 \\
0.001\end{array}$ & $\begin{array}{l}0.0276 \\
0.0779 \\
0.1274 \\
0.1746 \\
0.2309 \\
0.2902\end{array}$ & $\begin{array}{l}\sim 36 \\
\sim 13 \\
\sim 8 \\
\sim 6 \\
\sim 4 \\
\sim 3.5\end{array}$ \\
\hline
\end{tabular}


Table 3 Breakdown of costings per delivered dose of vaccine (from Mangtani et $\mathrm{al}^{7}$ )

\begin{tabular}{lll}
\hline Item & $\begin{array}{l}\text { Mass infant } \\
\text { vaccination }\end{array}$ & $\begin{array}{l}\text { GU clinic } \\
\text { vaccination } \\
(£)\end{array}$ \\
\hline Vaccine dose & $(£)$ & 9.82 \\
Staff cost per dose* & 7.36 & 4.20 \\
Syringe cost per doset & 1.40 & 0.03 \\
Needle per doset & - & 0.01 \\
Indirect cost to patient for attending for doses 2 and 3ł & - & 12.50 \\
Screening test & - & 5.00 \\
\hline
\end{tabular}

* Staff costs were estimated using 1993 salary scales.

† Assumption was made that vaccine for mass infant programme would be integrated with other infant vaccines by means of a combined preparation.

$\ddagger$ First dose given opportunistically, estimate of the equivalent of 1 hour of time for adult patient for each of sunbsequent doses.

Table 4 Estimated cost per patient used in this work

\begin{tabular}{lcc}
\hline & $\begin{array}{c}\text { Mass infant } \\
\text { vaccination }\end{array}$ & $\begin{array}{c}\text { GU clinic } \\
\text { vaccination }\end{array}$ \\
\hline Proportion receiving first dose who go on to receive second dose & 1.0 & $0.8^{*}$ \\
Proportion receiving first dose who go on to receive third dose & 1.0 & $0.6^{*}$ \\
Mean number of doses per patient & 3.0 & 2.4 \\
Estimated mean cost per patient (without screening) & $£ 26.28$ & $£ 51.25$ \\
Estimated mean cost per patient (with screening) & - & $£ 56.25$ \\
\hline
\end{tabular}

* Gilson, personal communication.

deed, in terms of lowering infection incidence in the population, the peak effect from a single year's vaccination may occur only after several decades. Because of this change over time in the community based effect of $\mathrm{HBV}$ vaccination after a programme has been started, cumulative totals are used to reflect the investment made throughout a programme or over a defined time period.

Data on monetary costs of delivering a course of vaccine ${ }^{7}$ have been used in conjunction with the measure used earlier ${ }^{8}$ (cumulative numbers of carriers prevented divided by cumulative numbers of doses), to provide an estimate of cost effectiveness in terms of cumulative numbers of carriers prevented per pound $(£)$ expenditure. A breakdown of these costings is given in tables 3 and 4 . Figure 2 (a) and (b) shows the cost effectiveness in combination with a simple measure of effectiveness. In these three dimensional figures, the higher the bars the more carriers prevented per $f$, and the "deeper" into the figure the more effective (more carriers prevented per year). The objective is a programme with a high cost effectiveness and a high measure of effectiveness. In considering the relationship between the two measures, the most desirable programme is the one in which the top of the bar is at the same time higher and "further" into the figure at a given point in time.

Discounting is often used when considering the effects of health interventions, but this is an area of considerable controversy. We present results in undiscounted form here, although there is no reason why this form of presentation should not prove equally suitable for analyses based on a discounting procedure.

\section{Results}

RELATIONSHIP BETWEEN EFFECTIVENESS AND COST EFFECTIVENESS

Previous work ${ }^{8}$ showed that increasing the vaccination rate resulted in a less than pro rata increase in effectiveness and that cost effectiveness also became slightly poorer as the vaccination level increased (these measures were about an order of magnitude less for heterosexuals than for homosexuals). Here it is assumed that $90 \%$ of the eligible population receives the first dose of a schedule in each case. Figure 2 shows the relationship between the cost effectiveness ratio and effectiveness for policies of universal vaccination of infants and vaccination targeted at attendees of $\mathrm{GU}$ clinics at time points of 10,25 , and 50 years after the start of vaccination. For infant vaccination it was assumed that all those given the first dose went on to receive the remainder of the course and that $99 \%$ became immune. ${ }^{14}$ For GU clinic attendees, of those receiving the first dose, $80 \%$ went on to receive the second dose and $60 \%$ the third (Gilson R, personal communication). Seroconversion rates for doses one, two, and three were set at $31 \%, 89 \%$, and $99 \%$ respectively for heterosexuals, and $24 \%, 77 \%$, and $92 \%$ respectively for homosexuals. ${ }^{15}$

Figure 2(a) shows results for the heterosexual population; the cost effectiveness of targeted vaccination is clearly much greater at all time points than for infant vaccination. In terms of effectiveness, $\mathrm{GU}$ vaccination has more impact over 10 and 25 year time spans (deeper into the page), but effectiveness of infant vaccination increases markedly over the second 25 years. It is nearly 40 years since the start of vaccination, however, before its effectiveness exceeds that of GU clinic vaccination

Figure 2(b) shows a somewhat different picture for homosexuals. The lower portions of the light bars show the effect of targeted vaccination without prior screening, assuming that $90 \%$ of individuals attending a GU clinic are vaccinated (unless vaccinated on a previous occasion with confirmation of a satisfactory vaccine response). In this case $\mathrm{GU}$ clinic vaccination is much more effective than universal infant vaccination at all time points as a continuing programme of infant vaccination will take several decades to have an impact on the high levels of sexual transmission compared with targeted vaccination across all age groups. The high rate of clinic attendance by homosexuals increases the efficiency of this mode of delivery, coupled with the impact on transmission of vaccinating those with high rates of partner change. Clinic vaccination also remains much more cost effective than mass infant vaccination at 10 and 25 years, but by 50 years the cost effectiveness of infant vaccination is better. The cost effectiveness advantage enjoyed by clinic vaccination is less enduring for homosexuals than for heterosexuals because of the high proportion of homosexuals who have already experienced infection (much vaccine is therefore wasted if prior screening is not carried out) and the higher partnership rates of the homosexual population in general.

The influence of prior screening on vaccination of homosexuals in GU clinics is illustrated by the combined upper and lower portions of the light bars. Overall effectiveness is of course unchanged, but cost effectiveness is roughly doubled, becoming much greater 

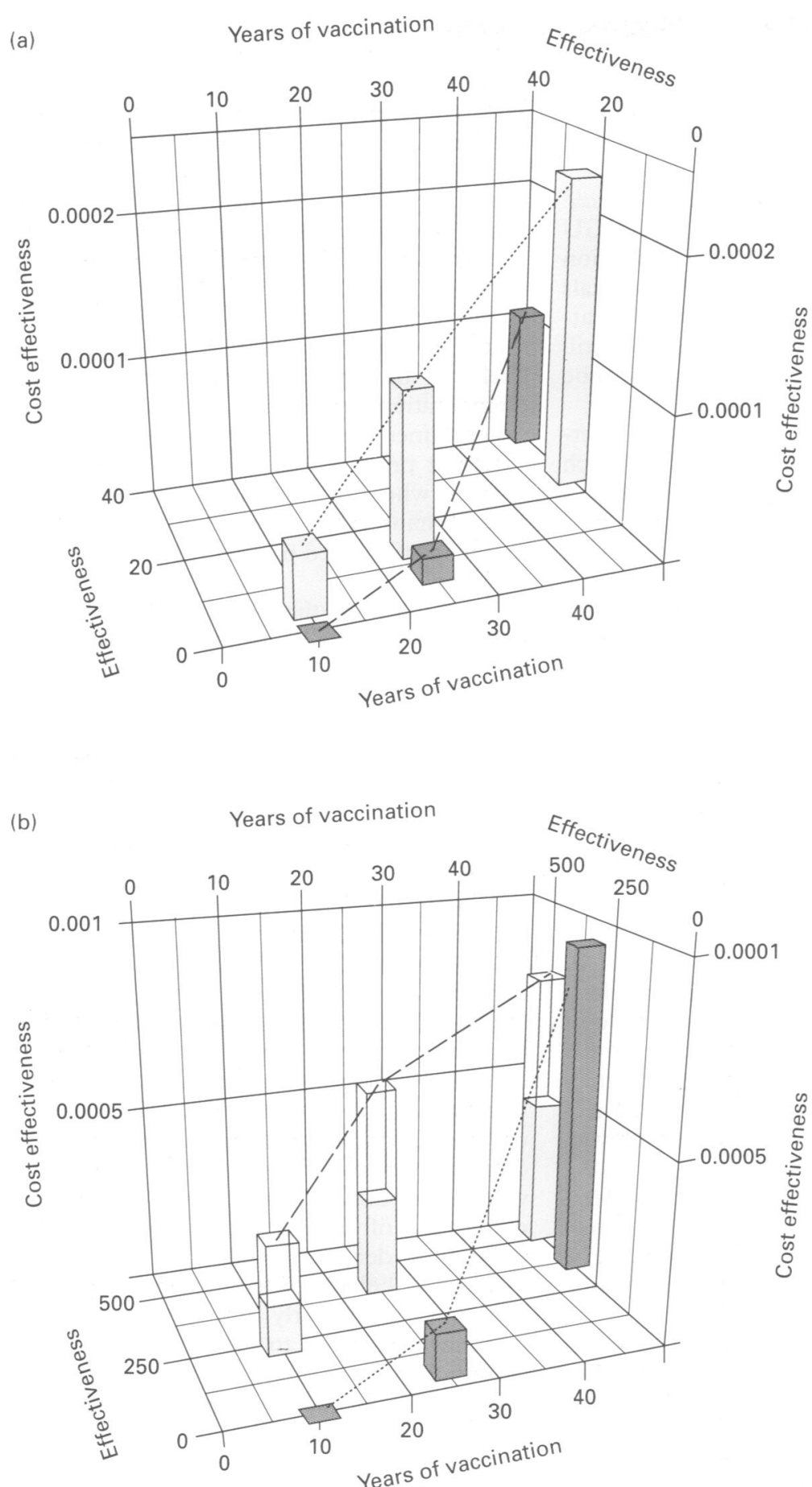

Figure 2 The relationship between cost effectiveness represented by cumulative numbers of carriers prevented per $f$ and effectiveness represented by the cumulative number of carriers prevented per year at time points 10, 25, and 50 years since the start of a vaccination programme in (a) heterosexuals and (b) homosexuals. Dark bars represent the effect of mass infant vaccination and light bars vaccination at genitourinary clinics. In graph (b) the lower portions of light bars correspond to clinic vaccination of homosexuals without prior screening and the upper and lower portions combined correspond to clinic vaccination after screening.

than for mass vaccination of infants for the first 25 years and only slightly less than that for infants after 50 years.

\section{Discussion}

Vaccine effectiveness may be defined in terms of the protection of the individual or by reference to the degree of success achieved in interrupting the chain of transmission (the cre- ation of herd immunity). The protection of the individual is a matter of clinical judgement (taking account where appropriate of relevant guidelines), but effectiveness in interrupting the chain of transmission is a public health issue and therefore relevant to the design of community or population based vaccination programmes. Effectiveness of this kind can be assessed by considering the numbers of cases of infection or disease prevented which would otherwise have occurred in the absence of mass targeted immunisation.

The effectiveness of a vaccination programme is quite different from its cost effectiveness. High levels of both are desirable, but a programme may be highly effective but have only a modest level of cost effectiveness. Alternatively it may be highly cost effective without being effective in terms of interrupting the transmission chain. An example is the vaccination of neonates born to infectious mothers. They are at very high risk of infection and of becoming carriers of $\mathrm{HBV},{ }^{16}$ but because they seem to comprise a relatively small proportion of births overall ${ }^{1718}$ and only a small proportion will have large numbers of sexual partners later in life, ${ }^{12}$ vaccinating them against $\mathrm{HBV}$ will not have a significant effect on transmission. ${ }^{8}$ The greater effectiveness of $\mathrm{GU}$ clinic vaccination lies in the fact that it has the potential for a disproportionately high impact on the transmission chain, but this potential depends on the effectiveness of the mechanism for delivering vaccine to those deemed most important in the spread of HBV infection. It therefore relies on the frequency of attendance at GU clinics by individuals of different sexual activity classes. This observation underlines the need for further surveys to quantify more precisely the relationship between rates of attendance and sex partner change rate. Audit work to establish levels of vaccination achieved by GU clinics in the $\mathrm{UK}$ is becoming more common, ${ }^{1019}$ but there is also a need to establish patterns of compliance in patients in relation to their age and partner change rate.

With this need for better data in mind and having assumed plausible rates of compliance and seroconversion for each of the three doses, the model results suggest that vaccination targeted at homosexuals attending GU clinics could be highly effective in stemming HBV transmission, and more so than mass infant immunisation. In making this observation, however, a word of caution is appropriate. Notifications to the Communicable Diseases Surveillance Centre (CDSC) of acute cases of HBV suggest some $24 \%$ of cases with identified risk factors are associated with intravenous drug use compared with $54 \%$ for sexual transmission. ${ }^{8}$ At present our model omits this additional age dependent transmission process and its relative contribution to overall levels of transmission remains uncertain. Vaccination of infants and those attending GU clinics will, however, affect levels of transmission between intravenous drug users by reducing the pool of HBV infection in the population. It is also true that there are uncertainties in the CDSC data. Nearly half of all reported cases have no risk 
factor identified, numbers of unreported asymptomatic infections are likely to be significant, ${ }^{20}$ and the level of misdiagnosis is unknown (it has been observed that in many cases symptoms can easily be mistaken for those of glandular fever for example ${ }^{21}$ ).

As was noted earlier and in Williams et al, ${ }^{8}$ a high proportion of homosexuals attend GU clinics relatively regularly. Targeted vaccination is somewhat less effective for heterosexuals because of their comparatively low rate of attendance at GU clinics, but nevertheless it still takes nearly 50 years for its effectiveness to be exceeded by mass immunisation of infants.

Over the longer term ( $>50$ years) the effectiveness of mass infant vaccination is much greater than that of targeted vaccination because of low rates of clinic attendance by heterosexuals. Because delivery occurs across all ages for GU programmes and because of the time it takes for vaccinated infants to reach a sexually active age (an argument in favour of vaccination in adolescence) vaccination in $\mathrm{GU}$ clinics will show its benefits earlier. However, our analyses suggest that the long term potential of targeted vaccination approaches that of infant vaccination, for the homosexual population at least. It may even be greater given present uncertainties regarding data. Vaccination compliance rates at GU clinics are a source of uncertainty and more data are needed, but available information suggests a high drop out rate between doses (Gilson R, personal communication). ${ }^{1019}$ Nevertheless, and bearing in mind that there would be cost implications, with a higher publicity profile for HBV vaccination and policies consistently applied it may well be possible to make significant improvements in this aspect of vaccination delivery.

Turning from effectiveness to cost effectiveness, we have discussed previously ${ }^{8}$ some of the many imponderables of this topic. The costings reported in Mangtani et $a l^{7}$ and used in these projections, include an estimate of the indirect cost to the patient of attending a GU clinic for the second and third doses of a course which accounts for over one third of the overall cost of clinic vaccination. The merits of including this cost element are open to debate, and figures shown here for cost effectiveness of GU clinic vaccination would increase by roughly one half if this were excluded from the calculation. Even with this element included, our projections show that $\mathrm{GU}$ clinic vaccination of heterosexuals can be much more cost effective than mass infant vaccination (fig $2(a)$ ). With screening before vaccination in GU clinics, clinic vaccination of homosexuals is also much more cost effective at 10 and 25 years and values of the cost effectiveness measure for infant and homosexual vaccination policies are broadly similar at 50 years (fig 2(b)).

Although better data are clearly needed, the strength of this modelling approach lies in the ease with which fresh projections can be carried out as and when improved data become available. It is also a very straightforward matter to test how sensitive the results of a vaccination programme are to variations in, for example, level of uptake $\mathrm{e}^{22}$ or changes in vaccine cost, by simply varying the appropriate input (using a more sophisticated but still straightforward sensitivity analysis, ${ }^{23}$ interactions between parameters can also be explored). We have used cost estimates based on UK conditions, but our model framework makes it a simple matter to provide revised projections relevant to other countries, given appropriate costings and parameter estimates. Although the cost of vaccine in many countries, including the UK, is such that effects of differences in delivery costs are likely to be small by comparison, the implementation of mass infant vaccination programmes occasions opportunities for negotiating price reductions for bulk supply in markets where the advent of a number of new recombinant $\mathrm{HBV}$ vaccines provides the potential for increased price competition between suppliers.

In conclusion, the view is often expressed that vaccination targeted at high risk groups has failed to reduce the $\mathrm{HBV}$ incidence. It should be borne in mind that $\mathrm{HBV}$ vaccination began only in the early 1980 s so that any measure of impact can be based on little more than a decade of transmission. The work reported here and in Williams $e t a l^{\beta}$ shows that the long duration of the carrier infectious period of HBV means it will be many years before any vaccination policy which is not aimed universally at all age groups has a major effect, and decades before its full impact is clear (underlining the importance of being clear about the time scale of an assessment of cost effectiveness). Moreover this work starts from the conservative assumption that incidence of new infection was at equilibrium prior to the start of vaccination. If incidence were actually increasing (for example, see data from the USA ${ }^{24}$ ) when vaccination was introduced it would take many years of vaccination before the incidence started to decline at all. In the meantime changes in behaviour as a result of the AIDS epidemic, particularly amongst homosexuals, may have occurred to complicate the picture further. It is clear, therefore, that at the present time we could not yet expect to have unequivocal evidence of the success or failure of a targeted (or indeed any) HBV vaccination programme.

Our comments should not be read as advocacy in favour of targeted programmes. They are simply intended to emphasise the fact that targeted programmes do have a potential that is not wholly appreciated and which may not be given full weight in any debate on the relative merits of universal and targeted programmes. In this context our observations underline the importance of an appreciation of the transmission dynamics of $\mathrm{HBV}$ to informed decisions about implementing vaccination programmes.

We wish to thank the Department of Health for their financial support of this work. DJN is supported by a Royal Society University Research Fellowship. We express our appreciation to the NATSSAL team for access to anonymous numerical data from the survey, and to Dr John Edmunds of the Department of Biological Sciences, University of Warwick for his advice. We gratefully acknowledge discussions with Dr Andrew Hall of the London School of Hygiene and Tropical Medicine, Dr Richard Gilson of University College Hospital, London, Pro- 
fessor Jonathan Weber, and Dr Primrose Watkins of St Mary's Hospital, London, Dr Julia Heptonstall of PHIS CDSC, London, Dr Elizabeth Boxall of Birmingham PHLS, and Drs Simon Barton and (he Centre for the Epidemiology of Infectious Disease, Oxford for their assistance and helpful comments. We also wish to thank Cambridge University Press for their permission to reproduce data in table 1 and BMJ Publications for their permission to reproduce par of table 3.

1 Ghendon Y. WHO strategy for the global elimination of new cases of hepatitis B. Vaccine 1990;8(Suppl 1):S129-S33.

2 Roure $C$ Overview of epidemiology and disease burden of hepatitis B in the European region. Vaccine 1990;8(Suppl 1):S18-S21:

3 Kattamis C, Papaevangelou G. Workshop group: Greece Vaccine 1990;8(Suppl 1):S97-S8.

4 Jilg W. Selective risk group strategies in Europe. Vaccine 1990;8(Suppl 1):S44-S6

5 Roure C. Congress objectives. Vaccine 1990;8(Suppl 1): S9-S10.

6 Kane $M$. Global programme for control of hepatitis B infection. Vaccine 1990;8(Suppl 1):S47-S9.

7 Mangtani P, Hall AJ, Normand CEM. Hepatitis B vaccination: the cost effectiveness of alternative strategies in England and Wales. 7 Epidemiol Community Health 1995; 49:238-44.

8 Williams JR, Nokes DJ, Medley GF, Anderson RM. The transmission dynamics of hepatitis B in the UK: a mathematical model for evaluating costs and effectiveness of immunisation programmes. Epidemiol Infect 1996;116(1): 71-89.

9 El-Dalil A, Radcliffe KW, Bailey J, Richmond RA, Wade AAH. A survey on hepatitis B vaccination policies in genitourinary medicine in UK and Ireland Genitourin Med 1995;71:251-3.

10 Bhatti N, Gilson RJC, Beecham M, Williams P, Matthew MP, Tedder RS, Weller IVD. Failure to deliver hepatitis $B$ vaccine: confessions from a genitourinary medicine clinic. $B M \mathcal{F}$ 1991;303:97-101.

11 Fine PEM. Herd immunity: history, theory and practice. Epidemiol Rev 1993;15(2):265-302.
12 Johnson AM, Wadsworth J, Wellings K, Field J, Bradshaw S. Sexual attitudes and lifestyles. Oxford: Blackwell Scientific Publications, 1994.

13 Anderson RM, May RM. Infectious diseases of humans: dynamics and control. Oxford: Oxford University Press, 1991.

14 André FE. Overview of a 5-year clinical experience with a yeast-derived hepatitis B vaccine. Vaccine 1990;8(Supp): S74-S8.

15 Laukamm-Josteb U, von Laer G, Feldmeier H, Bienzle U, Uy A, Thomssen R, Guggenmoos-Holzmann I. Active immunization against hepatitis B: Immunogenicity of a recombinant DNA vaccine in females, heterosexual and homosexual males. Postgrad Med f 1987;63(Supp2):14346. 16 Wheeley SM, Tarlow MJ, Boxall EH. Chronic hepatitis B
in male and female children of $\mathrm{HBsAg}$ carrier mothers $\mathcal{f}$ Hepatol 1989;8:226-31.

17 Brook MG, Lever AML, Kelly D, Rutter D, Trompeter RS, Griffiths P, Thomas HC. Antenatal screening for hepatitis $B$ is medically and economically effective in the hepatitis B is medically and economically effective in the prevention of vertical transmission: three years
in a London hospital. $O 7 M$ 1989;71:313-17.

18 Derso A, Boxall EH, Tarlow MJ, Flewett TH. Transmission of HBsAg from mother to infant in four ethnic groups. BMF 1978,1:949-52.

19 Nandwani R, Barton S. Medical audit of hepatitis B immunization policy in a genitourinary medicine clinic. Int f STD AIDS 1993;4:244.

20 Sherlock S. Hepatitis B: the disease. Vaccine 1990,8(Suppl 1):S6-S9.

21 Clee WB, Hunter PR. Hepatitis B virus in general practice Epidemiology, clinical and serological features, and control. $B M F 1$ 1987;295:530-2.

22 Williams JR Nokes DJ, Anderson RM Assessment of the impact of various strategies to control hepatitis $B$ infection. In: Zuckerman $\mathrm{A}$ ed. The prevention of hepatitis $B$ in the In: Zuckerman A ed. The prevention of hepd
newborn, children and adolescents. In press.

newborn, children and adolescents. In press.
23 Blower SM, Dowlatabadi H. Sensitivity and uncertainty analysis of complex models of disease transmission: an HIV model, as an example. International Statistical Review 1994;62(2):229-43.

24 Kane M. Epidemiology of hepatitis B infection in North America. Vaccine 1990;8(Suppl 1):S16-S17. 